\title{
Development of fast scattering model of complex shape target for seminatural tests of onboard proximity radars in real time mode
}

\author{
Andrei K. Likhoedenko ${ }^{1}$, Victor B. Suchkov ${ }^{2}$ \\ ${ }^{1}$ Higher School of Economics, Department of applied mathematics, 101000, ul. Myasnitskaya, 20, Moscow, Russia \\ ${ }^{2}$ Bauman Moscow State Technical University, Department of autonomous information and control systems, 105005, ul. Baumanskaya 2-ya, \\ 5/1, Moscow, Russia
}

\begin{abstract}
Problems of creation of models of real time of complex shape targets on the basis of use of their polygonal models are considered. Formulas for radar cross section of multipoint model of target and power of input signal of onboard radar are described. Technique of semi-natural tests of onboard radar detector on the base of multipoint model of target is proposed. Results of digital simulation of input signals of the onboard radar detector of the target from the aerodynamic target on the basis of their multipoint models are given.
\end{abstract}

\section{Introduction}

For simulation and efficiency estimation of onboard proximity radar detectors of targets semi-natural tests are widespread amongst researchers and institutes [1-2]. Semi-natural tests of proximity inboard radars can be implemented on the base of hardware stand with channel of target signal imitation. In case of semi-natural tests for onboard radar detectors imitation of target signal is carried out by calculation of scattering electromagnetic fields from complex shape targets in near zone of radarlocation [3]. Such type of calculation could be developed on the base of exact electrodynamic methods such as method of moments (MoM), Multilevel Fast Multipole Method (MLFMM).

But in case of electrically large size target it would be possible to apply Physical Optics (PO) [3] with Physical Theory of Diffraction (PTD) on the base of polygonal model of target [4]. But the polygonal model is practically unsuitable for fast estimation of the operation of onboard radar during its tests at semi-natural stand because of huge computer resources of calculation of targets with large number of polygonal elements even with use of parallel algorithms [5]. So it is necessary to develop fast scattering model of target for operative seminatural tests and efficiency estimation.

\section{Technique of creation of fast scattering model of the target}

For creation of fast scattering model of the target we can use its polygonal model. As one of advanced CAD system for creation of complex shape target we can use PTC Creo Parametric. It is possible to create target with high level of complexity of target surface with taking into account of edges in Creo Parametric. Some examples of polygonal models of targets created in Creo Parametric are shown at Figure 1 and Figure 2.

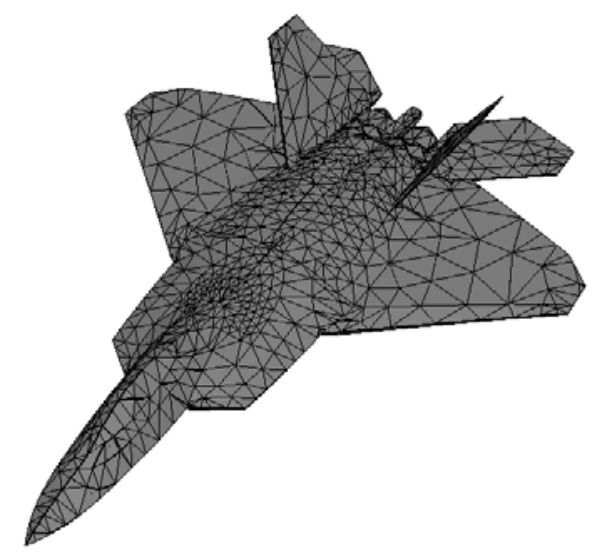

Figure 1. Polygonal model of fighter F-22

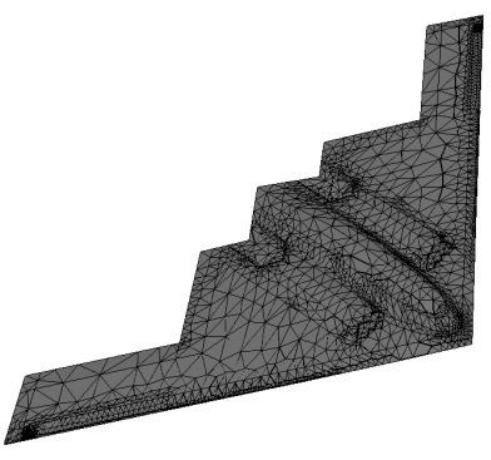

Figure 2. Polygonal model of bomber B-2 
Resulting intensity of the scattered electromagnetic field from complex shape target on the base of its polygonal model:

$$
\dot{E}_{\text {scat } \Sigma}=\sum_{m=0}^{N_{\Sigma}-1} \dot{E}_{s m}=\sqrt{\frac{P_{i} D_{i} Z_{0}}{2 \pi}} \sum_{m=0}^{N_{\Sigma}-1} F_{i m} \dot{\rho}_{s m} \frac{\exp \left(-\mathrm{i} k R_{i m}\right)}{R_{i m} R_{s m}},
$$

where $\mathrm{P}_{\mathrm{i}}$ is the power of the signal radiated by the transmitting system, $W ; D_{i}$ is the directive gain of the transmitting antenna in the direction of the maximum radiation; $Z 0=120 \pi$ is the wave impedance of air, $\Omega$; $\mathrm{R}_{\mathrm{i}(\mathrm{s}) \mathrm{m}}$ is the distance between the phase center of the antenna and reflector, $\mathrm{m} ; \mathrm{F}_{\mathrm{i}(\mathrm{s})}$ is the value of the directivity function of the transmitting antenna in the direction toward the reflector; $\dot{\rho}_{i m}^{s}$ is the complex reflection coefficient of the reflector, $m$.

For creation of fast scattering model of the target we can use multipoint target which can be formed on the base of calculation of scattering fields from local parts of polygonal model. Thus polygonal model of the target is divided in different cubes. Each cube limits specified part of target surface (Figure 3).

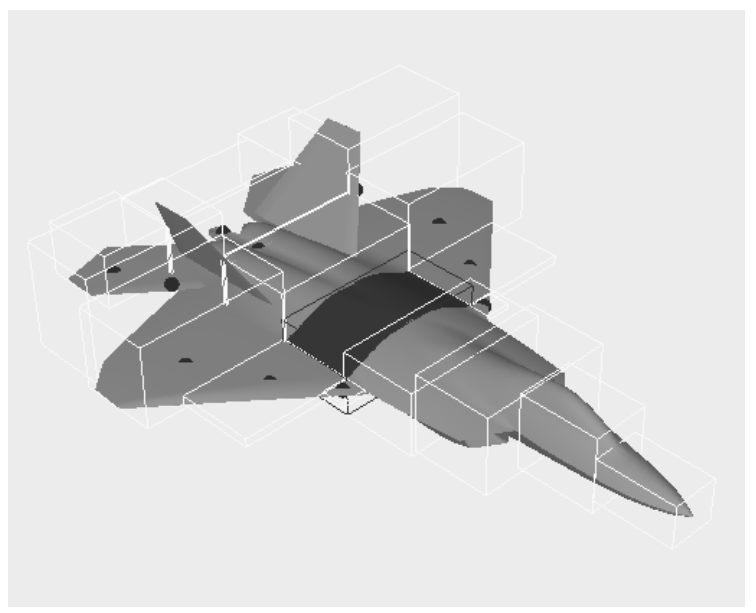

Figure 3. Formation of multipoint model of fighter F-22 on the base of local cubes

Description of elementary reflectors of polygonal model is based with use of local points $P_{n}=\left\{\begin{array}{lll}X_{n} & Y_{n} & Z_{n}\end{array}\right\}$ and triangles $T_{k}=\left\{\begin{array}{llll}P_{1 k} & P_{2 k} & P_{3 k} & N_{k}\end{array}\right\}$ (Figure 1). For formation of the multipoint model array of the local cubes $\Psi_{k}$ of specified sizes have to be created. Each cube limits specified part of target. Furthermore, we can form array of triangles of polygonal model $T_{k j}$ which specified cube includes.

As a result, for each m-th cube there will correspond the "brilliant" point. Thus multipoint model of the target consists of array of "brilliant" points each of them will correspond surface equivalent of complex target:

$$
P_{m}=\left\{\begin{array}{lll}
X_{m} & Y_{m} & Z_{m}
\end{array}\right\}, m=1 . . N_{m},
$$

where $m$ - number of brilliant point, $N_{m}=N_{c u b}$ - number of brilliant points (cubes).

For each cube of target (brilliant point) diagram of radar cross section (RCS) have to be calculated in the local coordinate system of brilliant point $X_{m}, Y_{m}, Z_{m}$
(Figure 4). Calculation of RCS could be carried out in azimuth and angles of vision range $\alpha_{0}=0, \alpha_{\max }=360^{\circ}$, $\beta_{0}=0, \beta_{\max }=360^{\circ}$, in the local coordinate system of brilliant point for specified distance to radar detector. RCS of target have to be calculated by PO and PTD methods on the base of formula (1) for scattering field of target on the base of its polygonal model. RCS of the local part of the target could be described on the base of known formula:

$$
\sigma^{m}(R, \alpha, \beta)=4 \pi R^{2} \frac{\left|\dot{E}_{\mathrm{s}}^{(\mathrm{m})}(R, \alpha, \beta)\right|^{2}}{\left|\dot{E}_{\mathrm{i}}^{(\mathrm{m})}(R, \alpha, \beta)\right|^{2}} .
$$

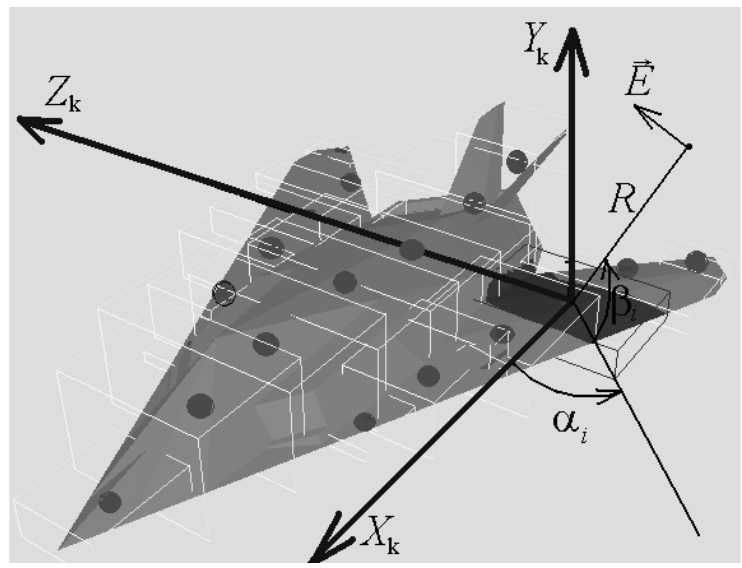

Figure 4. Formation of local coordinate system of local cube

As a result, for each brilliant point of the target array of RCS in local coordinate system $\sigma_{i, j}=\sigma\left(\alpha_{i}, \beta_{j}\right)$ will be formed. Multipoint model of fighter F-22 with surface diagram of RCS one of point on the distance $\mathrm{R}=5 \mathrm{~m}$ from radar detector is shown at Figure 5.

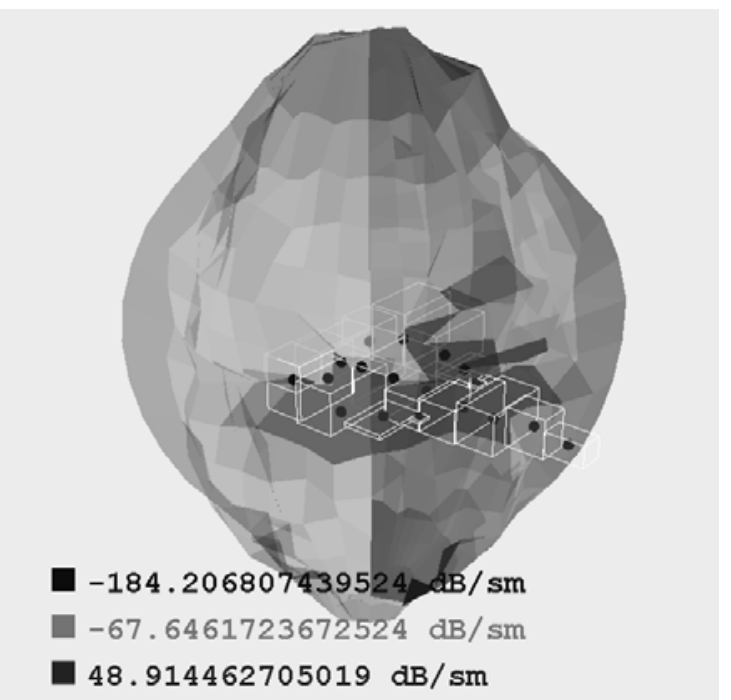

Figure 5. Multipoint model of fighter F-22 and RCS diagram one of its point

Each RCS array have to be saved in text file for specified brilliant point. So number of text files with RCS diagrams of brilliant points are the source data for calculation input signal of onboard radar detector at seminatural tests. 


\section{Technique of semi-natural tests of onboard radar detector on the base of multipoint target}

For calculation of input signals of onboard radar detector from target we have to describe the scheme of mutual position of onboard radar and the target (Figure 6).

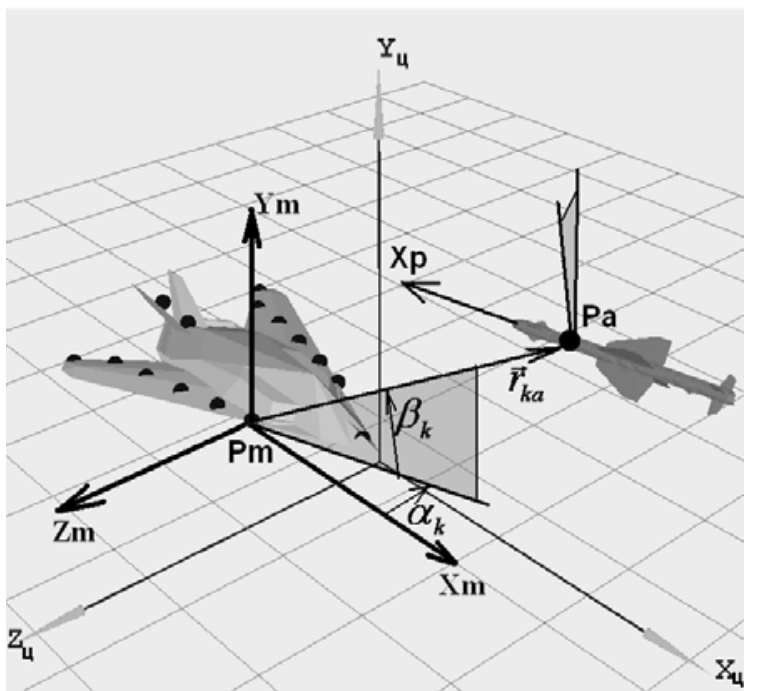

Figure 6. The scheme of mutual position between target and onboard radar

Trajectory of target in coordinate system of onboard radar is direct line from start point of target $P_{t 0}=\left\{x_{t 0}, y_{t 0}, z_{t 0}\right\}$ to last point of target $P_{t k}=\left\{x_{t k}, y_{t k}, z_{t k}\right\}$. For each position of the target we can calculate the power of input signal of target with use of arrays of RCS of each brilliant point. So power of the input signal from target for specified position of target on trajectory of its movement could be obtained by formula:

$$
\begin{aligned}
& P_{r e f l .}=\frac{P_{i} D_{i} D_{s} \lambda^{2}}{(4 \pi)^{2}}\left|\sum_{k=1}^{N_{m}} \frac{F_{i k} F_{s k} \dot{\rho}_{m}}{R_{m}^{2}}\right|^{2}, \\
& \dot{\rho}_{m}=\sqrt{\frac{\sigma_{m}}{4 \pi}} \exp \left(-i 2 \frac{2 \pi}{\lambda} R_{m}\right),
\end{aligned}
$$

where $P_{\text {refl }}$ is the power of the signal reflected from target, $\mathrm{W} ; \mathrm{D}_{\mathrm{s}}$ is the directive gain of the receiving antenna in the direction of the maximum radiation; $R_{m}$ is the distance between the phase center of the antenna and brilliant point, $\mathrm{m} ; \mathrm{F}_{\mathrm{i}(\mathrm{s})}$ is the value of the directivity function of the transmitting (receiving) antenna in the direction toward the brilliant point; $\dot{\rho}_{m}$ is the complex reflection coefficient of the brilliant point, $\mathrm{m} ; \sigma_{m}$ is RCS of the brilliant point of target from multipoint model, $\mathrm{m}^{2}$.

As an example the dependence of power of input signal of onboard radar detector from fighter F-22 at the trajectory of its movement is shown on Figure 7. Dependence of input power was calculated for typical onboard radar detector which operates at frequency $\mathrm{f}=10$ GHz. Moreover, the scheme of mutual position between fighter F-22 and onboard radar with antenna pattern is also shown at Figure 7.

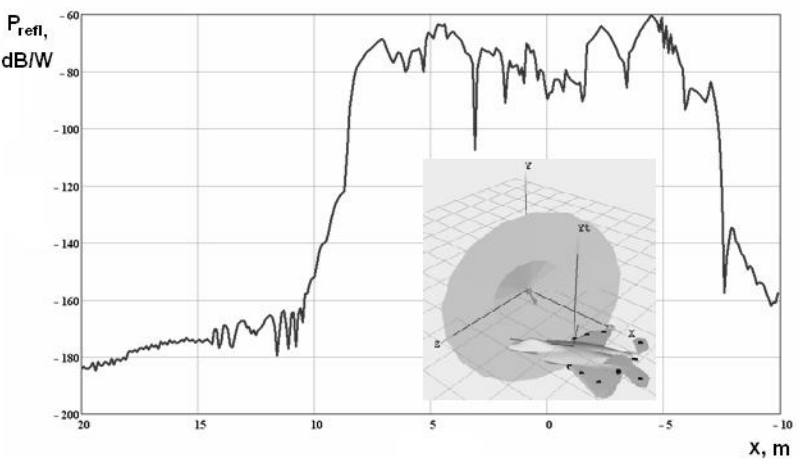

Figure 7. Dependence of power from fighter F-22 from coordinate $\mathrm{x}$

Calculation of power of reflected signal from fighter F-22 on the base of its multipoint model (Figure 7) has taken not more than $1 \mathrm{sec}$. So multipoint model of complex shape target (like at the Figure 5) could be considered as a fast scattering model of target. Developed multipoint model of the target could be applied in seminatural tests of onboard proximity radar detector.

For each point of target's multipoint model the following parameters could be defined:

- power of reflected signal from specified $m$-th brilliant point of target:

$$
P_{\text {refl. }(m)}=\frac{P_{i} D_{i} D_{s} \lambda^{2}}{(4 \pi)^{2}}\left|\frac{F_{i k} F_{s k} \dot{\rho}_{m}}{R_{m}^{2}}\right|^{2}
$$

- time delay of reflected signal from $\mathrm{m}$-th brilliant point:

$$
\tau_{m}=2 R_{m} / c ;
$$

where $R_{m}$ is distance between onboard radar detector and $\mathrm{m}$-th point.

So for each brilliant point of the target attenuation by voltage of generating signal from transmitter of onboard radar detector could be defined as following:

$$
\gamma_{m}=\sqrt{\frac{P_{r e f l(m)}}{P_{i}}},
$$

where $P_{i}$ - amplitude of power from output of generator, W.

On the base of formulas (5-7) input signal of onboard radar detector from target on the base its multipoint model could be defined as:

$$
U_{\text {inp }}(\mathrm{t})=\sum_{k=1}^{n} u_{\text {gen }}\left(\mathrm{t}-\tau_{\mathrm{m}}\right) \cdot \gamma_{m},
$$

where $n$ - number of points of target; $u_{g e n}(\mathrm{t})$ - voltage from output of generator of radar, $V$.

With use of definition of input signal of radar from complex target (8) on the base of multipoint model of target the functional scheme of stand for semi-natural tests of onboard radar is shown at Figure 8. So the stand for semi-natural tests have to consist of onboard radar detector, imitator of the target reflected signal and computer with special software for control of operation of the radar detector.

Imitator of target reflected signal is developed for simulation of real physical signal from target in real time mode in laboratory by creation of attenuation and time 
delay of generator signal. Operation of imitator is realized with use of control device and special software. Thus imitator consists of the following elements:

- operating dynamic time delay line;

- electrically operating attenuator in real time mode.

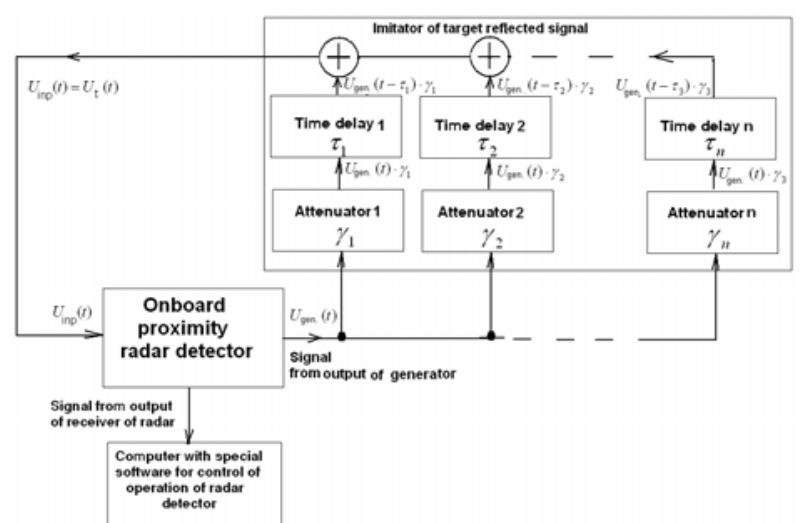

Figure 8. The functional scheme of stand for semi-natural tests of onboard proximity radar detector

The functional scheme of stand (Figure 8) could be applied for operative estimation of efficiency of onboard radar detector in real time mode for widespread mutual positions between target and onboard radar detector.

\section{Conclusion}

In this paper we have shown that high speed of calculation of input signals of onboard radar detector from complex shape targets could be achieved by use of multipoint model of target. To investigate availability of creation of fast scattering model, the general formulas of RCS of each specified local part of target corresponding to brilliant point of fast scattering model are developed. The results of calculation of power from fighter F-22 on the base of its multipoint model show that process of calculation has taken not more than $1 \mathrm{sec}$. So multipoint model of complex shape target could be considered as a fast scattering model of target with very high speed of calculation. From the research results, it is seen that the values of power of input signal of onboard radar could be applied for establishing of attenuations in the functional scheme of the stand of semi-natural tests.

\section{References}

1. Yin Jun, Deng Jia-hao, Wang Wei, 3 ICCEA Proceedings (Beijing, 2004)

2. Xiangjun Li, Jing Li, Tie Li, 10 ISAPE Proceedings (Xian, 2012)

3. A. B. Borzov, V. B. Suchkov, B. I. Shakhtarin, Yu. A. Sidorkina, J. of Comm. Tech. and Electr., 59, 13 (2014) 4. V. V. Akhiyarov, A. B. Borzov, V. B. Suchkov, B. I. Shakhtarin Yu. A. Sidorkina, J. of Comm. Tech. and Electr., 60, 8 (2015)

5. V. B. Suchkov, M. I. Baev, A. K. Likhoedenko, 5

IWCSE Proceedings (Moscow, 2015) 